\title{
Utilizing Multiple Home Links in Mobile IPv6
}

\author{
Hongbo Shi and Shigeki Goto \\ Department of Computer Science, Waseda University \\ 3-4-1 Ohkubo Shijuku-ku, Tokyo, 169-8555 JAPAN \\ Email: \{shi, goto\}@goto.info.waseda.ac.jp
}

\begin{abstract}
This paper proposes an improved mechanism for keeping stable connections between mobile nodes and corresponding nodes in the mobile IPv6 protocol. The mobile IPv6 protocol enables mobile nodes to keep the reachability while they are moving freely in the Internet. Our new method has multiple home links instead of one single home link in the current specification of the mobile IPv6 protocol.

The new method is based on our earlier idea of having multiple home agents. Multiple home agents can provide backups when a home agent is not working properly. However, all the home agents should reside on one single network segment according to the current specification of the mobile IPv6. This paper further extends our earlier idea of multiple home agents, and proposes multiple home links.

We have more stable connections by using multiple home links. This paper also shows a working example which illustrates the merit of multiple home links.
\end{abstract}

\section{INTRODUCTION}

This paper proposes a new idea in the protocol of mobile IPv6 [1]. The new idea enables a mobile node to have multiple home links at any time. The structure is shown in Fig. 1.

We have already proposed to have multiple home agents [2], which are shown as Home Agent 1 and Home Agent 2 in Fig. 1. Although Home Agent 1 and Home Agent 2 can work as a backup to each other, the reliability is limited because they reside on the same network segment, Home Link 1, in Fig. 1.

This paper extends the earlier idea and proposes to have multiple home links, Home Link 1 and Home Link 2, in Fig. 1. We will explain how to extend the protocol to realize this idea.

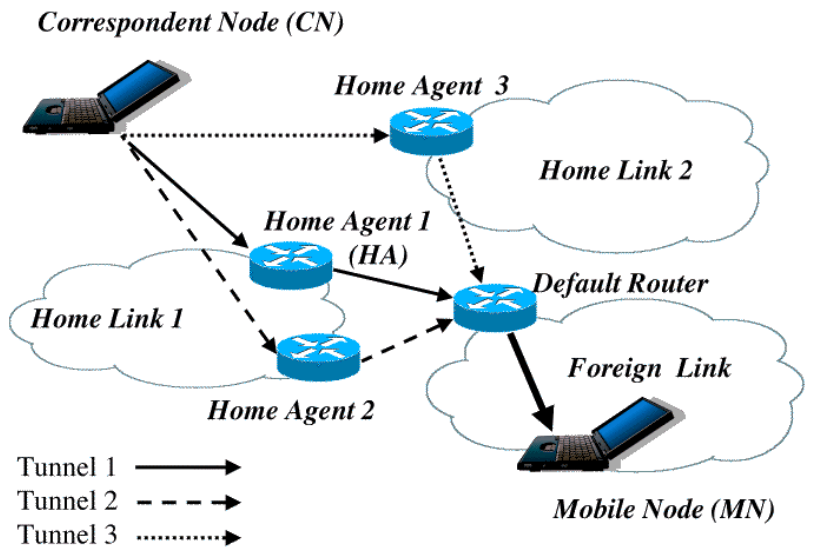

Fig. 1. Multiple Home Links Mechanism
The mobile IP protocol is a specification at the IP layer. It allows a mobile node to keep the connectivity while it is moving around in the Internet. Each mobile node has its home address as the identifier. When a mobile node is away from its home link, it is associated with a care-of address. The care-of address indicates the current foreign location of the mobile node. Mobile IPv6 is a well-known protocol which has been much improved from the original Mobile IPv4 protocol in RFC 3344 [3].

In Mobile IPv6, each mobile node has one single home link. And each mobile node has one single home agent on its home link. A Home Link is also called a home network. A Home Agent performs as a router on the home link which forwards packets to the mobile node when it is away from the home link.

Section II explains the basic mechanism of the existing Mobile IP. In Section III, we introduce our idea of Multiple Home Agents which improve the reliability of Mobile IP. Section IV proposes a new idea of Multiple Home Links. Section V gives a working example and Section VI concludes this paper.

\section{Basic Mechanism of Mobile IP}

A mobile node can keep the connections while it is moving freely in the Internet. Each mobile node has a home address as the unique identifier of the current location. It takes the form of a routable unicast IP address. This address belongs to the home link of the mobile node. When a mobile node moves away from its home link and locates at a foreign link, it needs a new IP address of the current location inside the foreign link. A foreign link is a network other than the home link of the mobile node, which is defined in the Mobile IPv6 protocol [1]. The new IP address is called a care-of address. In order to keep the network connection, a mobile node should register the current care-of address at the home agent immediately. Then, the home agent can forward packets bound for the home address to the mobile node. It makes a tunnel to the registered care-of address of the mobile node.

If a mobile node is located at the home link, packets to the home address are routed to the home link of the mobile node. Then, it simply follows the conventional routing mechanism.

When a mobile node moves to a foreign link and away from the home link, one or more care-of addresses may be associated with the mobile node. There are several ways to get a new IP address at the new foreign link. For example, it is possible to use the IPv6 stateless or stateful auto-configuration 
mechanism. After obtaining the IP address, the mobile node needs to register a new care-of address at the home agent which is located on the home link. This registration is called Home Registration (HR). The association between the home address and the care-of address is called a binding. By the use of the binding information, packets bound for the home address of a mobile node can be encapsulated and tunneled to the current care-of address of the mobile node by the home agent. When the lifetime of a binding is expired, the mobile node has to update it. This process is called Binding Update (BU).

In Mobile IPv6, the concept of foreign agents does not exist any more. A mobile node can operate in any foreign locations with no special support from the local router.

A home agent works as a router in the home link of a mobile node. In Mobile IPv6, if a correspondent node has the valid binding information of a mobile node, then it can send the packet directly to the care-of address of the mobile node. In this case, those packets do not go through the home agent. All the packets are routed directly to the router on the foreign link.

\section{Multiple Home Agents}

This paper is based on the idea of multiple home agents (Fig. 2), which has been proposed by the authors earlier [2]. This section explains the mechanism of multiple home agents.

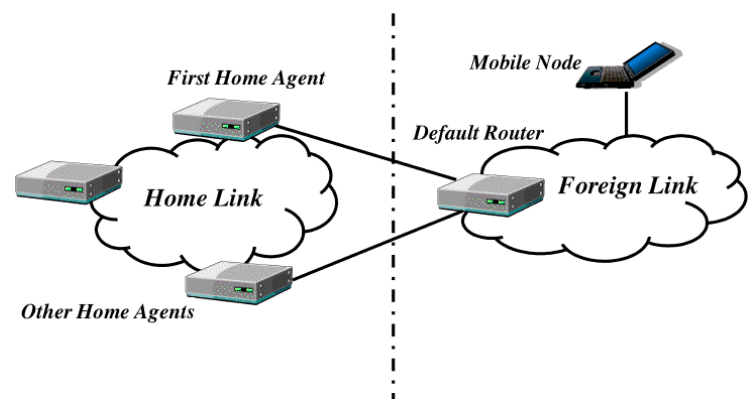

Fig. 2. Multiple Home Agents Mechanism

The current specification of Mobile IPv6 is described in the Internet Draft [1]. There are two significant problems in Mobility Support in IPv6 version 24 [1]. The first one is solved by our multiple home agents [2]. The second problem can be solved by our new proposal suggested in Section IV which is called multiple home links.

The first serious problem is described as follows. When a home agent is out of order, nobody can contact a mobile node until the mobile node finds a new home agent. According to the Mobile IPv6 Internet Draft, a mobile node does not know if its home agent is dead or alive in a short time when the mobile node is located at foreign links. A mobile node can find the home agent is down only when it updates the binding information at the home agent or when it moves to a new link.

The idea of multiple home agents allows a mobile node to have more than one home agents on the home link when it is away from home. If one home agent is out of order, other remaining home agents can forward the packets to the mobile node (Fig. 3). For simplicity, it is assumed that a mobile node is not multi-homed in this paper. If a mobile node has two IP addresses due to multi-homing, it can have two home agents at the same time. This paper does not deal with such a case.

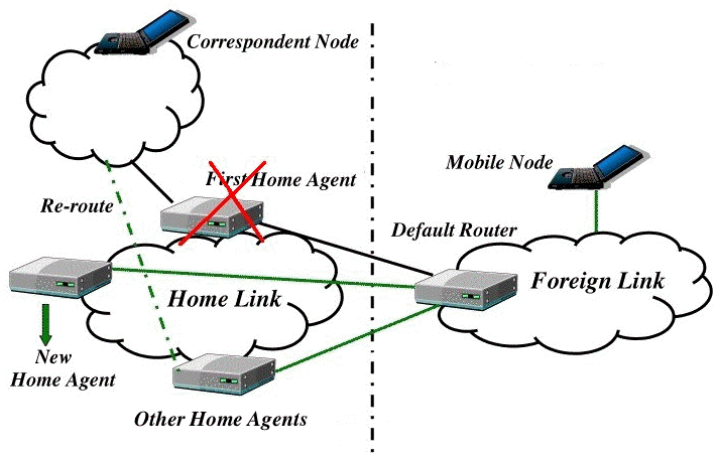

Fig. 3. Routing to another HA

\section{A. Home Agent Unreachability Detection}

A group of home agents maintains the home agent lists, through the use of Neighbor Unreachability Detection among home agents [4].

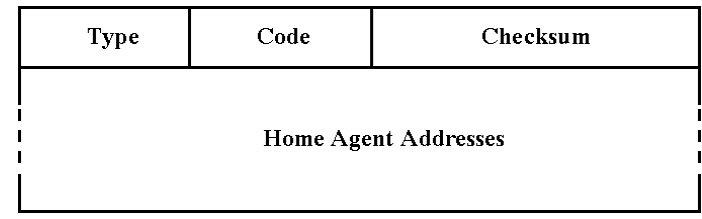

Fig. 4. ICMP Home Agent Unreachable Error

In Mobile IPv6, Home Agent Unreachability Detection is used to know if other home agents which serves to the same mobile node on the same home link is properly working. Home Agent Unreachability Detection can check if any other home agents are alive or dead. If a home agent finds that some other home agents which serve to the same mobile node are unreachable, the home agent will specify the IP addresses of the unreachable home agents to the ICMP Unreachablity Message to inform the mobile node. When the mobile node receives this message, it starts to find another new home agent on its home link immediately. If a mobile node can find a new home agent, it starts using a new agent.

In Mobile IPv6, there are four new ICMP messages. They are Home Agent Address Discovery Request, Home Agent Address Discovery Reply, Mobile Prefix Solicitation and Mobile Prefix Advertisement. In our method, a new ICMP message is proposed. It is Home Agent Unreachability Error shown in Fig. 4 [2].

In Fig. 4, the Home Agent Address Field is used to specify the IP addresses of unreachable home agents. Type value is set to 154 temporarily in this paper. ${ }^{1}$

\footnotetext{
${ }^{1}$ The value should be fixed by IANA/ICANN.
} 


\section{B. Modified Binding Update}

In Mobile IPv6, when a mobile node moves from a link to another link, the mobile node is assigned a new care-of address from the new foreign link. The mobile node needs to update the binding information cached at the home agent as well as at the correspondent nodes.

Each home agent should keep the correct binding information. Our new method recommends the mobile node to update the information at all the home agents at once.

In our new method, Binding Update Message is modified from the original specification of [4]. It is used to inform that Multiple Home Agents Mechanism is in use. Fig. 5 shows that a new $\mathrm{M}$ bit is added in the Binding Update Message.

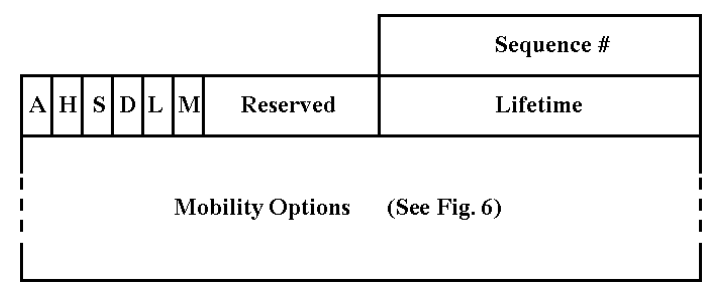

Fig. 5. Modified Binding Update Message

When $M$ bit is set, it means that the mobile node is using Multiple Home Agents Mechanism. Then, new options including multiple Home Agents Addresses (Fig. 6) can be used. Through the M bit, the home agent knows there are other home agents which serves to the same mobile node. The home agents can get the IP addresses of other home agents from the option field of this message.

There is a function called Dynamic Home Agent Discovery used in the Mobile IPv6 protocol [1]. In order to perform this function, each home agent needs to provide a Home Agents List [1] which keeps the information about all the home agents at the same link. Each home agent responds to a query sent by a mobile node with the home agent list.

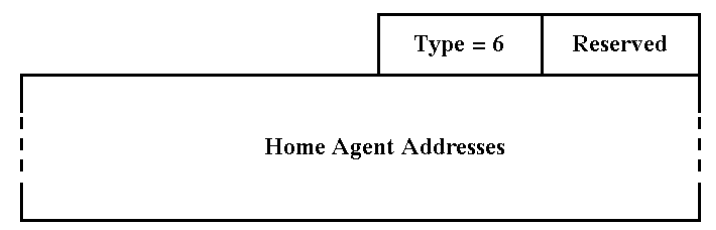

Fig. 6. Multiple Home Agents Option

A home agent which uses Multiple Home Agents Mechanism should check the IP addresses listed in the Multiple Home Agents Option field with the current Home Agents List which should be kept at the home agent. If the home agent cannot find an IP address of one of the current home agents in the Option field, the home agent should send an ICMP Home Agent Unreachable Error Message described in Sec. III-A to the mobile node. The mobile node will not use the home agent specified by the ICMP error message.

\section{New Proposal - Multiple Home Links}

This paper proposes a new mechanism called Multiple Home Links. This idea already appeared in Fig. 1.

In the current specification of Mobile IPv6 [1], a mobile node can have one single home link for a network interface. According to this specification, a mobile node can have only one home agent on the home link.

If the home link is not reachable by some reason, the mobile node loses its connections with corresponding nodes who do not keep the binding information. Typically, a mobile node cannot initiate a new connection even if the foreign link is working without any trouble.

This paper proposes the use of multiple home links to solve the problem. As shown in Fig. 7, when one of home links of a mobile node is disconnected from the Internet, a correspondent node can use another home address which is assigned at Home Link 3 to communicate with the mobile node.

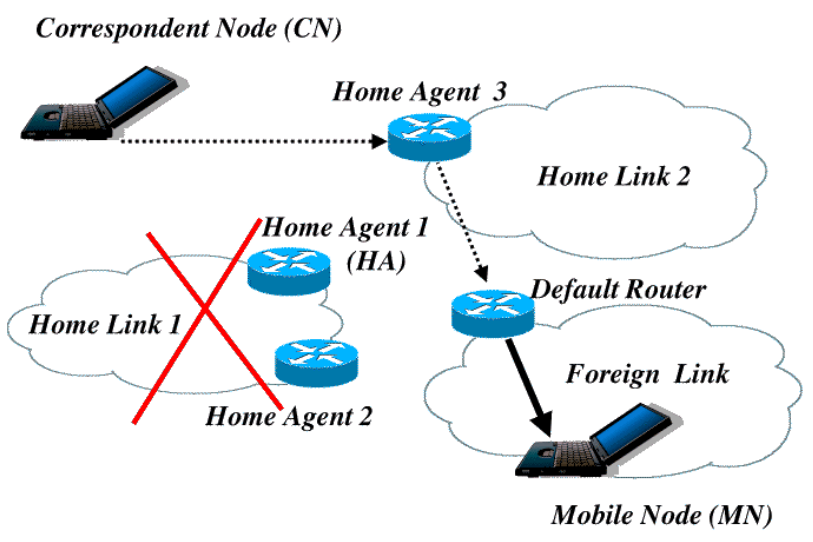

Fig. 7. Change Route to Another Home Link

\section{A. Modifications in Binding Update Procedure}

The new Multiple Home Links mechanism is an extension of our earlier proposal of Multiple Home Agents (Section III). The Multiple Home Links mechanism allows a mobile node to have multiple home agents at different links, not limited to one single link. This extension provides variety of routes towards a mobile node no matter where the mobile node is. When a home link is out of order, the packets sent to the mobile node can be transferred by other home agents at other reachable home links.

In the current specification of Mobile IPv6, a mobile node only has to send the binding update messages to the home agent or to the correspondent nodes when the mobile node is away from home. In order to realize the new mechanism, the binding update procedure is modified. In this paper, when a mobile node is at a home link, the mobile node still has to send binding update messages to the home agents which are at different home links from the mobile node. When a mobile node is at a home link, if there is any correspondent nodes which use other home addresses of the mobile node with different network prefix from the current home link, it has to send binding update messages to the correspondent nodes. 


\section{B. State Transition}

This paper proposes a new method which enables a mobile node to have multiple home agents at different links. This subsection describes how the new method works. We use the following classification of movements.

- Initial State: A mobile node is at one of the home links.

- Move 1: A mobile node moves from a home link to anther home link.

- Move 2: A mobile node moves from a home link to a foreign link.

- Move 3: A mobile node moves between foreign links.

- Move 4: A mobile node moves back to one of the home links from a foreign link.

A mobile node has to send binding registration messages or binding update messages to the home agents which are at the different links from the mobile node.

In the current Mobile IPv6 protocol, the Movement Detection mechanism is used. Movement Detection is performed by a combination of IPv6 Neighbor Discovery, Router Discovery and Neighbor Unreachability Detection. By looking at the subnet prefix of the assigned care-of address, we can tell whether a mobile node moved and changed the attached link.

When a mobile node is at the Initial State, the mobile node has to find at least one home agent at each home link and send the Home Registration Messages to those home agents which are at different home links from the current link where the mobile node is located.

When a mobile node is at one of the home links, the home address which is assigned from the current attached home link is regarded as a care-of address of the mobile node by other home agents which are at the different home links.

When a mobile node makes Move 1, the mobile node has to send Home Registration Messages to those home agents which are at different home links from the current attached link. At the same time, the mobile node discards the old binding information of the home agent at the old home link.

Both in the Initial State and in Move 1, the mobile node should send binding update messages to the correspondent nodes who are using other home addresses. The mobile node should discard old binding information on the correspondent nodes who are using the home address that assigned from the current attached home link.

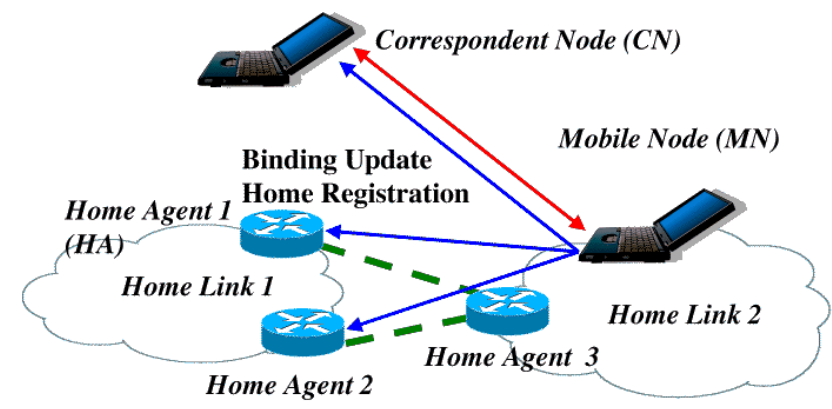

Fig. 8. Send Binding Update Messages When $\mathrm{MN}$ is on a Home Link

It should be noted here that all the home agents can be found by using Dynamic Home Agent Address Discovery protocol
[1]. A mobile node sends an ICMP Home Agent Address Discovery Request Message to the Mobile IPv6 home agents by using the anycast address for the home IP subnet prefix. When a home agent receives this message, the home agent should return a Home Agent Address Reply Message including the Home Agents List to the mobile node. The mobile node choose at least one home agent from the list. Then, the mobile node sends Home Registration Messages to the multiple home agents and waits for the Binding Acknowledgement from the home agents.

In Mobile IPv6, a mobile node maintains all the binding information as a Binding Update List. When a mobile node start using the Multiple Home Agents Mechanism, the mobile node put a new entry to the Bind Update List.

When a mobile node has multiple home agents, it sends Binding Update Message with the Multiple Home Agents Option (shown in Fig. 6) to the home agents.

When a mobile node makes Move 2 or Move 3, the mobile node obtains a new care-of address from the new foreign link. Then, it sends the Binding Update Messages to all the nodes listed in the Binding Update List.

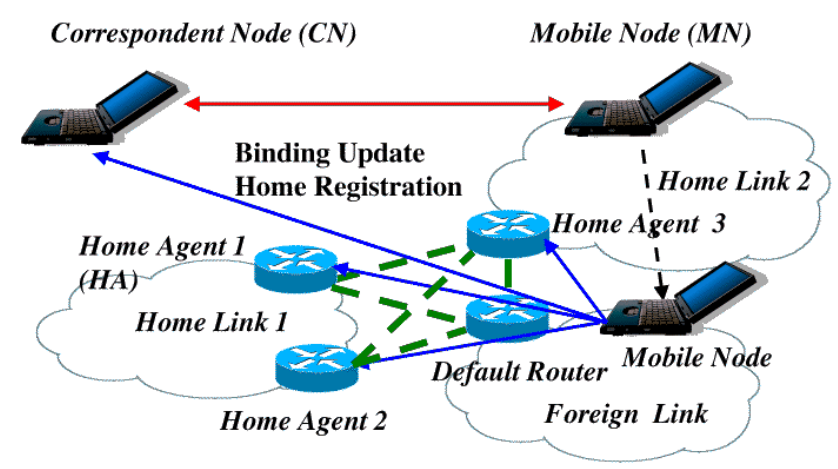

Fig. 9. Send Binding Update Messages When $\mathrm{MN}$ is on a Foreign Link

When a mobile node makes Move 4, the mobile node performs a similar function to Move 1 .

\section{IMPLEMENTATION AND EXPERIMENTS}

We have implemented the essential part of the new multiple home agent functions. It is a modification of KAME IPv6 distribution [5]. KAME has the function described in the Internet Draft, Mobility Support in IPv6 version 24 [1]. In our implementation, a mobile node has multiple home agents at multiple home links.

We use a testbed shown in Fig. 10. There are two home agents, Home Agent 1 and 2, on the home link 2001:200:1:2::/64 (Home Link 1). There is Home Agent 3 at the home link 2001:200:1:4::/64 (Home Link 2). There are two foreign links, Foreign Link 1 has IP address 2001:200:1:3::/64 and Foreign Link 2 has IP address 2001:200:1:6::/64. A mobile node moves between its home links and the foreign links in the testbed.

A mobile node can have multiple home addresses by using our Multiple Home Links Mechanism. There are several ways for a correspondent node to deal with multiple home addresses 


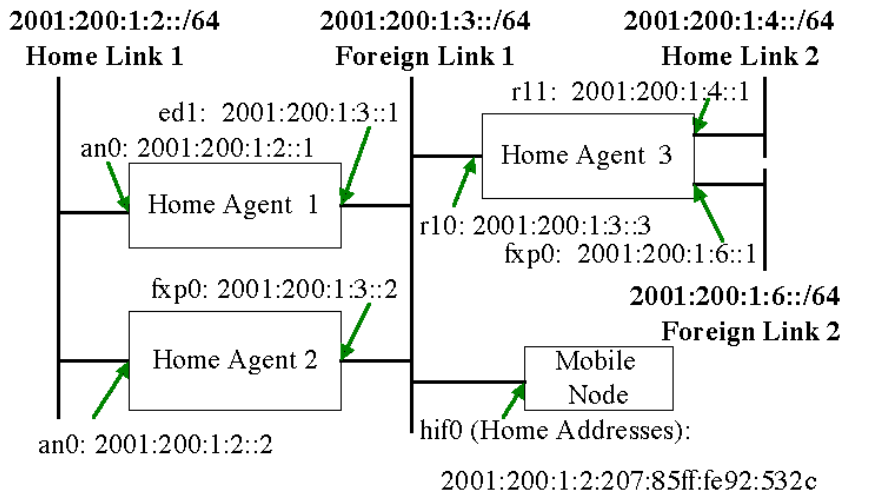

2001:200:1:4:207:85ff:fe $92: 532 \mathrm{c}$ an0 (Care-of Address):

2001:200:1:3:207:85ff:fe $92: 532 \mathrm{c}$

Fig. 10. Multiple Home Links Testbed

of a mobile node. A simple way is to use Domain Name System (DNS) [7] to convert the domain name of a mobile node to multiple home addresses in IPv6.

In our testbed, we have a DNS server which maintains two different home addresses of a mobile node. Fig. 11 shows the DNS zone file in our testbed.

There is a well-known mechanism in DNS, which is called round robin [8]. Using round robin, DNS server returns a different IP address every time it receives a request. In the current experiment, the DNS server returns alternative home address to each request.

When a correspondent node does not have a binding cache of a mobile node, all the packets must be forwarded by the home agent. If the home agent is out of order or the home link is not reachable, the correspondent node loses the connection with the mobile node. The mobile node also loses the connection with the correspondent nodes.

Fig. 12 shows the first experiment. We turn off Home Link 1 and send ping (ICMP ECHO messages) to the mobile node from the correspondent node. We specify the domain name of the mobile node. In Fig. 12, the correspondent node can keep the connection with the mobile node because of IPv6 Route Optimization [1] which does not use the home agent.

Fig. 13 shows the second result. We use mip6control with the option - $\mathrm{C}$ to delete the binding cache of mobile-gw which is a correspondent node. mip6control is a built-in command of KAME. After erasing the binding cache, we send the same ping messages to the mobile node. In Fig. 13, the correspondent node without valid binding cache cannot keep the connection with the mobile node. The connection is gone.

The third experiment is shown in Fig. 14. By using Multiple Home Links mechanism, the mobile node has another working home link and the home address. Since DNS works in round robin, the DNS server returns the second home address to the correspondent node. The correspondent node uses another home address and establishes the connection with the mobile node as shown in Fig. 14.

Our modified KAME implementation allows a mobile node to have multiple home agents on multiple home links. In the current Mobile IPv6 protocol, a mobile node will lose the connections to those nodes which do not have the binding information. The problem is fixed by the new method of multiple home links.

By using our modified implementation, when a mobile node is away from home, it sends the binding information to Home Agent 1, Home Agent 2 and Home Agent 3 at the same time. Home Agent 1, Home Agent 2 and Home Agent 3 have the same binding cache of the same mobile node. If the Home Link 1 is down, the correspondent node keeps the connection even if it does not have the valid binding cache of the mobile node. It can utilize another reachable home address assigned from Home Link 2.

\section{Conclusion and Future Plan}

According to the current specification of Mobile IPv6 in the Internet Draft, Mobility Support in IPv6, if the home link is out of order, the mobile node will lose its connections with those correspondent nodes who do not keep the binding cache of the mobile node. The mobile node cannot solve this problem using the current protocol.

This paper proposed a new method for a mobile node to have multiple home links. It also means that a mobile node can have multiple home agents at different home links. Even if one of home links is not reachable or dead, the mobile node still can use remaining home agents at reachable home links to keep forwarding packets to the mobile node.

We have implemented the functions, including finding multiple home agents at different home links, sending Binding Update and Registration Messages to multiple home agents at different home links.

In the future, we will investigate the binding update message to enable a correspondent nodes to control the connection with multiple home addresses. When a home links is down, the correspondent node can change the home address to a workable one during the communication.

\section{REFERENCES}

[1] D. Johnson, C. Perkins and J. Arkko, "Mobility Support in Mobile IPv6", draft-ietf-mobileip-ipv6-19.txt, Work in progress, October 29 2002. (The latest version is draft-ietf-mobile-ipv6-24.txt)

[2] H. Shi and S. Goto, "Multiple Home Agents in IPv6 networks", 65th annual Convention of IPSJ (Information Processing Society Japan), 5T95, pp.5-107-5-110, March, 2003. (In Japanese)

[3] C. Perkins, "IP Mobility Support for IPv4", RFC 3344, IETF, August 2002.

[4] T. Narten et. al., "Neighbor Discovery for IP Version 6 (IPv6)", RFC 2461, IETF, December 1998.

[5] Wide Kame Project, http://www.kame.net.

[6] S. Deering, "ICMP Router Discovery Messages", RFC 1256, IETF, September 1991.

[7] P. Mockapetris, "DOMAIN NAMES - CONCEPTS AND FACILITIES", IETF, November 1987.

[8] T. Brisco, "DNS Support for Load Balancing”, RFC 1794, IETF, April 1995.

[9] G. Malkin, R. Minnear, "RIPng for IPv6", RFC 2080, IETF, January 1997.

[10] C. Perkins, “IP Encapsulation within IP”, RFC 2003, IETF, October 1996.

[11] S. Knight et. al., "Virtual Router Redundancy Protocol”, RFC 2338, IETF, April 1998. 
Fig. 11. AAAA records on DNS zone file

mobile-gw\# ping mobile-node.mip6.goto.info.waseda.ac.jp

PING6 (56=40+8+8 bytes) 2001:200:1:4:240:96ff:fe37:1fb3 - > 2001:200:1:2:207:85ff:fe92:532c

16 bytes from 2001:200:1:2:207:85ff:fe92:532c, icmp_seq=0 hlim=64 time=4.325 ms

16 bytes from 2001:200:1:2:207:85ff:fe92:532c, icmp_seq=1 hlim=64 time=4.468 ms

16 bytes from 2001:200:1:2:207:85ff:fe92:532c, icmp_seq=2 hlim=64 time=4.036 ms

16 bytes from 2001:200:1:2:207:85ff:fe92:532c, icmp_seq=3 hlim=64 time=3.921 ms

16 bytes from 2001:200:1:2:207:85ff:fe92:532c, icmp_seq=4 hlim=64 time=3.938 ms ${ }^{\wedge} \mathrm{C}$

- - mobile-node.mip6.goto.info.waseda.ac.jp ping6 statistics - -

5 packets transmitted, 5 packets received, $0 \%$ packet loss

round-trip $\mathrm{min} / \mathrm{avg} / \mathrm{max} / \mathrm{std}-\mathrm{dev}=3.921 / 4.138 / 4.468 / 0.220 \mathrm{~ms}$

Fig. 12. PING (1) Home Address 1 with Binding Cache

mobile-gw\# mip6control - C 2001:200:1:2:207:85ff:fe92:532c

mobile-gw\# Oct 5 01:57:33 mobile-gw/kernel: .././netinet6/mip6_binding.c:2267

: BC timer stopped.

mobile-gw\# ping mobile-node.mip6.goto.info.waseda.ac.jp

PING6 (56=40+8+8 bytes) 2001:200:1:4:240:96ff:fe37:1fb3 - -> 2001:200:1:2:207:85ff:fe92:532c

${ }^{\circ} \mathrm{C}$

- - mobile-node.mip6.goto.info.waseda.ac.jp ping6 statistics -. -

8 packets transmitted, 0 packets received, 100\% packet loss

Fig. 13. PING (2) Home Address 1, after erasing the binding cache

mobile-gw\# ping mobile-node.mip6.goto.info.waseda.ac.jp

PING6 (56=40+8+8 bytes) 2001:200:1:4:240:96ff:fe37:1fb3 - -> 2001:200:1:4:207:85ff:fe92:532c

16 bytes from 2001:200:1:4:207:85ff:fe92:532c, icmp_seq=0 hlim=64 time=3.989 ms

16 bytes from 2001:200:1:4:207:85ff:fe92:532c, icmp_seq=1 hlim=64 time=3.634 ms ${ }^{\circ} \mathrm{C}$

- - mobile-node.mip6.goto.info.waseda.ac.jp ping6 statistics -. -

2 packets transmitted, 2 packets received, $0 \%$ packet loss

round-trip $\mathrm{min} / \mathrm{avg} / \mathrm{max} / \mathrm{std}-\mathrm{dev}=3.634 / 3.811 / 3.989 / 0.178 \mathrm{~ms}$

Fig. 14. PING (3) Selecting Home Address 2 by Multiple Home Links

[12] W. Richard Stevens, TCP/IP Illustrated, Volume 1: The Protocols, Addison-Wesley, 1994.

[13] W. Richard Stevens, TCP/IP Illustrated, Volume 2: The Implementation, Addison-Wesley, 1995.

[14] W. Richard Stevens, UNIX Network Programming, Volume 1, Second Edition: Networking APIs: Sockets and XTI, Prentice Hall, 1998.

[15] W. Richard Stevens, UNIX Network Programming, Volume 2, Second Edition: Interprocess Communications, Prentice Hall, 1999. 